\title{
Ultrafast dynamic computed tomography myelography for the precise identification of high-flow cerebrospinal fluid leaks caused by spiculated spinal osteophytes
}

\author{
Kent R. Thielen, MD, ${ }^{1}$ John C. Sillery, MD, ${ }^{2}$ Jonathan M. Morris, MD, ${ }^{1}$ Joseph M. Hoxworth, MD, ${ }^{3}$ \\ Felix E. Diehn, MD, ${ }^{1}$ John T. Wald, MD, ${ }^{1}$ Richard E. Rosebrock, MD, ${ }^{4}$ Lifeng Yu, PhD, ${ }^{1}$ and \\ Patrick H. Luetmer, MD'
} 1Department of Radiology, Mayo Clinic, Rochester, Minnesota; '2Department of Radiology, Billings Clinic, Billings, Montana;
3Department of Radiology, Mayo Clinic, Phoenix, Arizona; and ${ }^{4}$ High Plains Radiological Association, Amarillo, Texas

\begin{abstract}
OBJECT Precise localization and understanding of the origin of spontaneous high-flow spinal CSF leaks is required prior to targeted treatment. This study demonstrates the utility of ultrafast dynamic CT myelography for the precise localization of high-flow CSF leaks caused by spiculated spinal osteophytes.
\end{abstract}

METHODS This study reports a series of 14 patients with high-flow CSF leaks caused by spiculated spinal osteophytes who underwent ultrafast dynamic CT myelography between March 2009 and December 2010. There were 10 male and 4 female patients, with an average age of 49 years (range 37-74 years). The value of ultrafast dynamic CT myelography in depicting the CSF leak site was qualitatively assessed.

RESULTS In all 14 patients, ultrafast dynamic CT myelography was technically successful at precisely demonstrating the site of the CSF leak, the causative spiculated osteophyte piercing the dura, and the relationship of the implicated osteophyte to adjacent structures. Leak sites included 3 cervical, 11 thoracic, and 0 lumbar levels, with $86 \%$ of the leaks occurring from C-5 to T-7. Information obtained from the ultrafast dynamic CT myelogram was considered useful in all treated CSF leaks.

CONCLUSIONS Spinal osteophytes piercing the dura are a more frequent cause of high-flow CSF leaks than previously recognized. Ultrafast dynamic CT myelography adds value beyond standard dynamic myelography or digital subtraction myelography in the diagnosis and anatomical characterization of high-flow spinal CSF leaks caused by these osteophytes. This information allows for appropriate planning for percutaneous or surgical treatment.

http://thejns.org/doi/abs/10.3171/2014.10.SPINE14209

KEY WORDS cerebrospinal fluid leak; disc protrusion; headache; low pressure; osteophyte; technique

$\mathrm{S}$ PONTANEOUs high-flow fast spinal CSF leaks represent a specific type of CSF leak. Patients with highflow fast spinal CSF leaks have significant debility related to low-pressure CSF syndrome or superficial siderosis of the CNS., ${ }^{4,5,8,9,11,12}$ Detection of the exact site of leakage is difficult in patients with high-flow fast CSF leaks because of the rapid equilibration of intrathecal contrast with the epidural fluid collection. The identification and localization of low-flow slow leaks requires different techniques. ${ }^{1}$ The presence of spinal extraarachnoid fluid on
MRI is associated with the presence of a fast CSF leak and should direct the myelographer to evaluate the patient using dynamic CT myelography. ${ }^{7}$ Magnetic resonance imaging, in its current state of technological development, does not provide the necessary temporal resolution required to pinpoint the exact site of a high-flow spinal CSF leak. Case reports have described possible associations between spinal CSF leaks and nerve root sleeve tears, arachnoid diverticula, spinal degenerative lesions, and calcified disc protrusions. . $^{2,5,6,10}$ 
Historically, conventional CT myelography with acquisition of spinal radiographic images followed by transfer of the patient to the CT scanner was used to identify whether the patient had a spinal CSF leak. These same images were also scrutinized in an attempt to identify the site and cause of the leak. This technique is limited by the time delay in transfer of the patient to the CT scanner. In the case of high-flow fast CSF leaks, the rapid equilibration of intrathecal contrast with the epidural fluid collection results in difficulty identifying the exact site of the leak. The dynamic CT myelography technique reported by Luetmer and Mokri described CT myelography technique modifications aimed at improving temporal resolution in an attempt to capture earlier images of the site of the leak following administration of myelographic contrast. ${ }^{6}$ Precise localization and anatomical delineation of the leak site remained challenging despite this modification in technique. In retrospect, this technique was not fast enough to identify the exact site of the leak in many patients with high-flow fast CSF leaks. The rapidity and speed with which high-flow CSF leaks occur was not fully recognized until the report by Hoxworth et al. described the localization of a rapid CSF leak utilizing digital subtraction (DS) myelography. ${ }^{3}$ That case report demonstrated the near-immediate leakage of myelographic contrast into the epidural space at the time of the DS myelography. It then became apparent that modification of the dynamic CT myelography technique to an ultrafast technique utilizing immediate imaging of the myelographic contrast administration into the spinal canal, along with an altered patient position similar to the DS myelography technique, may provide increased opportunity to identify not only the site of high-flow fast CSF leaks, but also the origin of the leaks. Ultrafast dynamic CT myelography combines the volumetric high-resolution capabilities of current fast CT technology with the immediate physiological visualization that is obtained with DS myelography, showing relationships between high-flow fast CSF leaks and adjacent anatomical structures.

Precise anatomical localization and understanding of the origin of fast high-flow spinal CSF leaks is required prior to targeted treatment with surgery or percutaneous methods such as a localized blood patch. This study demonstrates the utility of ultrafast dynamic CT myelography for the precise identification and localization of high-flow spinal CSF leaks when caused by spiculated osteophytes originating from the spine.

\section{Methods}

\section{Study Sample}

This study was approved by the Mayo Clinic institutional review board. This retrospective study reports a series of 14 patients at Mayo Clinic in Rochester, Minnesota, with high-flow CSF leaks who underwent ultrafast dynamic CT myelography between March 2009 and December 2010. There were 10 male and 4 female patients, with an average age of 49 years (range 37-74 years). Each of these patients had either a prior conventional CT myelogram with an identified high-flow spinal CSF leak of indeterminate origin, or a head and spine MRI examination with extensive, multilevel, spinal ventral epidural fluid collection. Six (43\%) of the 14 patients had superficial siderosis on the craniospinal MRI examinations. Eight (57\%) of the 14 patients had findings of intracranial hypotension on craniospinal MRI examinations. In each of these cases high-flow CSF leaks, which appear to be caused by spiculated osteophytes originating from the posterior margin of the disc annulus, were precisely identified as the leak site utilizing the ultrafast dynamic CT myelography technique. Direct comparison with DS myelography was not possible because none of the patients included in this study underwent DS myelography. Over the time period encompassed in this study, there were 5 additional instances in 5 separate patients in which an ultrafast dynamic myelogram was completed. In 3 of these cases no active leak was identified, whereas in the other 2 cases a leak was identified that was believed to represent an iatrogenic leak at the site of a prior needle placement. These 5 cases were excluded from the study.

\section{Ultrafast Dynamic Myelography Technique}

In all cases the following ultrafast dynamic myelography technique was used. Lumbar puncture was completed with placement of a spinal needle under fluoroscopic guidance or on the CT scanner utilizing CT guidance. Care was taken to make sure the ventral spinal dura was not penetrated during the lumbar puncture. Ideally, the puncture is made away from any known collections of extradural fluid, to avoid confusing an epidural injection with spillage of contrast from the subarachnoid space into the extradural fluid. The patient was then positioned in a prone Trendelenburg position in the gantry of a 128-slice dual-source CT scanner (Siemens Medical Solutions). The patient's hips were elevated on a large wedge-shaped foam pad well above the chest. The shoulders were placed flat on the table, with the head extended slightly to hinder the flow of contrast into the cranial cisterns. Arms are ideally placed above the head to minimize attenuation of the x-ray beam, but may be placed at the sides as necessary for patient comfort. A lateral scout image was used to visualize the entire spine to ensure the free downward (cephalad) flow of myelographic contrast within the subarachnoid space and thus avoid pooling of the contrast within any dependent curves of the thoracic spine. Additional blankets or pillows were added below the hips as necessary to ensure adequate elevation of the patient's hips. Limited CT slices were obtained through the tip of the spinal needle to confirm needle location within the center of the spinal canal. On occasion a very small amount of contrast $(<0.5 \mathrm{ml})$ was injected with an immediate small group of images through the needle tip to document subarachnoid needle tip position.

A noncontrast CT scan of the spine was then obtained to serve essentially as a volumetric scout image. Ultrafast CT myelography was then performed with simultaneous slow hand injection of undiluted myelographic contrast (12.0 ml of Omnipaque 180, GE Health Care Ireland) into the thecal sac. Myelographic contrast is denser than CSF; therefore, the contrast will seek a dependent position rela- 
tive to CSF and thus flow rapidly downhill in a column of CSF while maintaining a dependent position within the subarachnoid space. The initial CT image acquisition was started simultaneously with the slow hand injection of myelographic contrast. This timing allowed for the nearcontinuous visualization of the leading edge of the bolus of myelographic contrast as it traveled in the subarachnoid space in a cranial direction from the needle tip to the foramen magnum. Care must be taken not to start the injection of contrast prior to the start of CT scanning. The myelographic contrast frequently leaks so briskly from the subarachnoid space that delay in the start of CT scanning may result in such extensive leak of contrast into the epidural space that the leak site cannot be precisely localized. The acquired images were rapidly processed and continuously viewed on a monitor in the CT scanner suite during the course of the myelographic contrast injection. Up to 6 serial alternating caudal-cranial and cranial-caudal CT acquisitions of the cervical, thoracic, and upper lumbar spine were obtained during the contrast injection. These acquisitions were prescribed by the $\mathrm{CT}$ technician prior to the contrast injection to minimize the delay between each acquisition. Minimal delay of up to 4.0 seconds occurred between each acquired series due to the inherent technical limits of the scanner. Once myelographic contrast was identified to be clearly leaking from the subarachnoid space, the CT technician was instructed to stop the image acquisition process. In most cases the presence of a fast leak could be identified within 3 series acquisitions. The needle was removed. The patient was returned to a flat prone position, log rolled, and then placed back on the scanner in a flat supine position. A single, approximately 5-minute, postcontrast injection delayed CT acquisition of the entire spine was then obtained. Effective dose per study was $70.64 \mathrm{mSv}$ (range 21.5-182.9 mSv). Although typically 3 or 4 scans are performed, as many as 6 scans could typically be performed immediately at $128 \times 0.6-$ $\mathrm{mm}$ collimation (1.0-second rotation time, $42.24 \mathrm{~mm} /$ rotation table feed, 1.1 pitch, $140 \mathrm{kV}$, and 208 quality reference $\mathrm{mAs}$ ) at the radiologist's discretion, with additional scans as necessary (either immediate or delayed). Automatic exposure control (CARE Dose 4D, Siemens Healthcare) was on so the radiation output was automatically adjusted according to the patient's size. The radiation dose for each acquisition is approximately $21.40 \mathrm{mGy}$ (CT dose index, or $\mathrm{CTDI}_{\mathrm{vol}}$ ). The helical data are initially reconstructed to $3.0-\mathrm{mm}$ section thickness at $3-\mathrm{mm}$ intervals by using a standard soft-tissue algorithm (window width 1000, window center 200 Hounsfield units) to allow rapid real-time assessment of caudocranial ascent of contrast within the spinal canal. Reconstructions can be performed at $0.6-\mathrm{mm}$ section thickness as desired. Curved sagittal and coronal reformats at $2.5-\mathrm{mm}$ section thickness at $2.5-\mathrm{mm}$ intervals are subsequently performed on all acquisition data sets. Total acquisition time for a 5-series study covering the cervical and thoracic spine was approximately 75 seconds.

\section{Image Review}

Reconstructed images were postprocessed and reviewed at an independent workstation, in conjunction with the axial source images. Multiplanar reconstructions with variable slice thicknesses were then scrutinized in detail by the neuroradiologist at the workstation. The leak location was identified by the appearance of a fork or split in the column of flowing myelographic contrast. The contrast flows down the ventral dependent portion of the spinal canal as a single column. When the leading edge of the contrast column encounters the leak site, a portion of the contrast flows through the hole in the dura into the ventral epidural fluid collection. This epidural contrast will then continue to flow downhill in a cranial direction. In addition, a significant portion of the contrast column in the subarachnoid space that has not escaped through the dural hole also continues to flow dependently downhill in a cranial direction as a second, somewhat linear-appearing column. The site of the split in the contrast column represents the leak location. Typically, the flow of contrast in the extrathecal region can be confirmed on subsequent acquisitions, with the development of homogeneous confluent increased attenuation of the collection of fluid on delayed images. The precontrast initial CT images were then also closely scrutinized at the location of the leak to determine if a small osteophyte was piercing or eroding the dura at the leak site.

The clinical records were reviewed for each patient to assess the utility of the information provided by the ultrafast dynamic CT myelogram.

\section{Results}

In 14 patients, ultrafast dynamic CT myelography was technically successful at precisely demonstrating the site of the high-flow fast CSF leak and the causative spiculated osteophyte piercing the dura, and the relationship of the implicated osteophyte to adjacent structures was identified. In each case, the ultrafast dynamic CT myelographic technique was successful in providing the temporal resolution needed to identify the CSF leak site prior to the diffuse spill of contrast into the epidural space, with subsequent obscuration of the exact site of the leak.

Leak sites included 3 cervical, 11 thoracic, and 0 lumbar levels (Fig. 1). Twelve (86\%) of the 14 leaks occurred from the C-5 to T-7 interspace levels. In each of the cases in this series there was a small spiculated osteophyte/calcified disc extrusion that had pierced or eroded through the dura, resulting in a high-flow fast CSF leak. These osteophytes all occurred at the level of the disc space in a midline or paracentral location along the dorsal margin of the disc annulus. In many cases the patients had multilevel osteophytes but only a single-level leak site. In no case was a large broad-based osteophyte observed to cause a leak.

There were no permanent procedural complications. A single patient had an approximately 1-minute episode of transient unresponsiveness that started shortly after the initiation of the myelographic contrast injection. This quickly resolved with removal of the spinal needle and return of the patient to a flat supine position. The leak location was localized from the images obtained.

Information obtained from the ultrafast dynamic CT 
myelograms was considered useful in all treated CSF leaks. The precise location of the leak relative to the adjacent osseous anatomy, such as the location relative to midline, was particularly helpful to the neurosurgeons in making treatment-planning decisions. In all surgically treated cases, the specific site of the leaking dural defect along with the implicated piercing spicule was visually apparent during surgery.

Ten $(71 \%)$ of the 14 patients underwent subsequent targeted therapeutic intervention directed at the leak site in the form of a targeted blood patch and/or surgery. Four patients (29\%) underwent no additional targeted intervention at our institution. Of the 10 patients with targeted intervention, $5(50 \%)$ were treated with a localized epidural blood patch, including a single patient who underwent a second localized blood patch. Seven patients (70\%) underwent surgery directed at repair of the dural defect. Two of these surgically treated patients underwent targeted blood patch placement at the level of the leak prior to surgery. Nine of the 10 patients treated with targeted intervention had subsequent follow-up ranging from 2 months to 12 months. All $9(100 \%)$ of these patients with follow-up had symptomatic and/or imaging improvement. Eight (89\%) of these 9 patients experienced symptomatic improvement. Of note, 2 of these patients demonstrated resolution of the extradural spinal fluid collection on follow-up MRI of the spine.

\section{Illustrative Cases}

\section{Case 1}

A 37-year-old otherwise healthy man presented with a 6-week history of relatively abrupt-onset, debilitating, orthostatic headaches and associated visual disturbances. The headaches were so severe that the patient preferred to spend most his waking hours supine. Contrast-enhanced MRI of the brain was obtained 9 days following the onset of the orthostatic headaches. Magnetic resonance imaging of the brain demonstrated mild generalized dural enhancement but was otherwise normal. MRI of the entire spine obtained 6 weeks following onset of the orthostatic headaches demonstrated a large, ventral, epidural fluid collection extending from the level of C-5 to the level of L-2, consistent with a spinal CSF leak. There were several scattered osteophytes identified in the cervical and thoracic spine. The next day a conventional CT myelogram was obtained that confirmed an extensive spinal CSF leak with extradural contrast filling a large ventral epidural fluid collection that extended from C-2 to T-7. The exact site of the leak could not be discerned on the conventional myelogram due to the diffuse extent of extradural contrast. Three days later the patient underwent an ultrafast dynamic CT myelogram that resulted in the precise localization of the leak, adjacent to a small spiculated osteophyte at the T2-3 interspace (Fig. 2). The next day the patient underwent a localized bilateral T2-3 CT-guided transforaminal epidural blood patch. The patient's headaches diminished for 1 week but then returned. The patient is contemplating further treatment with surgical intervention at this time.

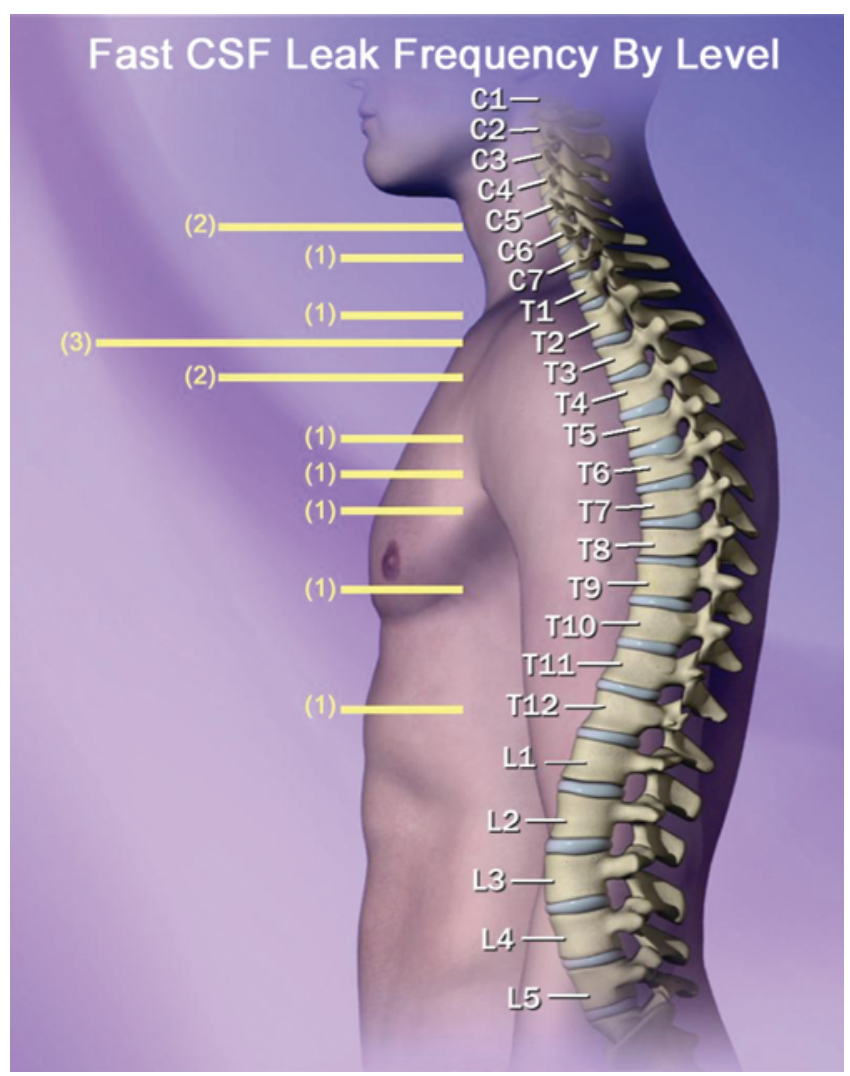

FIG. 1. Frequency of high-flow fast CSF leaks by spinal level. Used with permission from the Mayo Foundation for Medical Education and Research.

\section{Case 2}

A 51-year-old woman presented with a 6-month history of relatively abrupt-onset postural headaches and neck discomfort. The headaches would completely resolve if she lay flat. Two months prior to evaluation at our institution the patient suffered bilateral cranial vertex subdural hematomas and brain sag with inferior descent of the cerebellar tonsils identified on MRI of the brain. The patient presented to our institution 1 month later. Repeat MRI of the brain demonstrated a reduction in the size of the small bilateral subdural hematomas and persistence of the brain sag, suggestive of low-pressure headache syndrome. A conventional CT myelogram of the entire spine was performed the next day and demonstrated an extensive CSF leak with ventral epidural contrast extending from T-3 to T-10. An ultrafast CT myelogram of the spine was performed 5 days later in an attempt to precisely localize the leak site. The ultrafast CT myelogram localized the leak to the T5-6 interspace where a sharp osteophyte or calcified disc fragment was puncturing the dura (Fig. 3). CT-guided bilateral T4-5 transforaminal localized epidural blood patches were placed later the same day. Blood patches were pursued at the level adjacent to the CSF leak due to improved access of needle placement. The patient reported complete resolution of her severe postural headaches. At 1 year following treatment her positional headaches have not returned. 

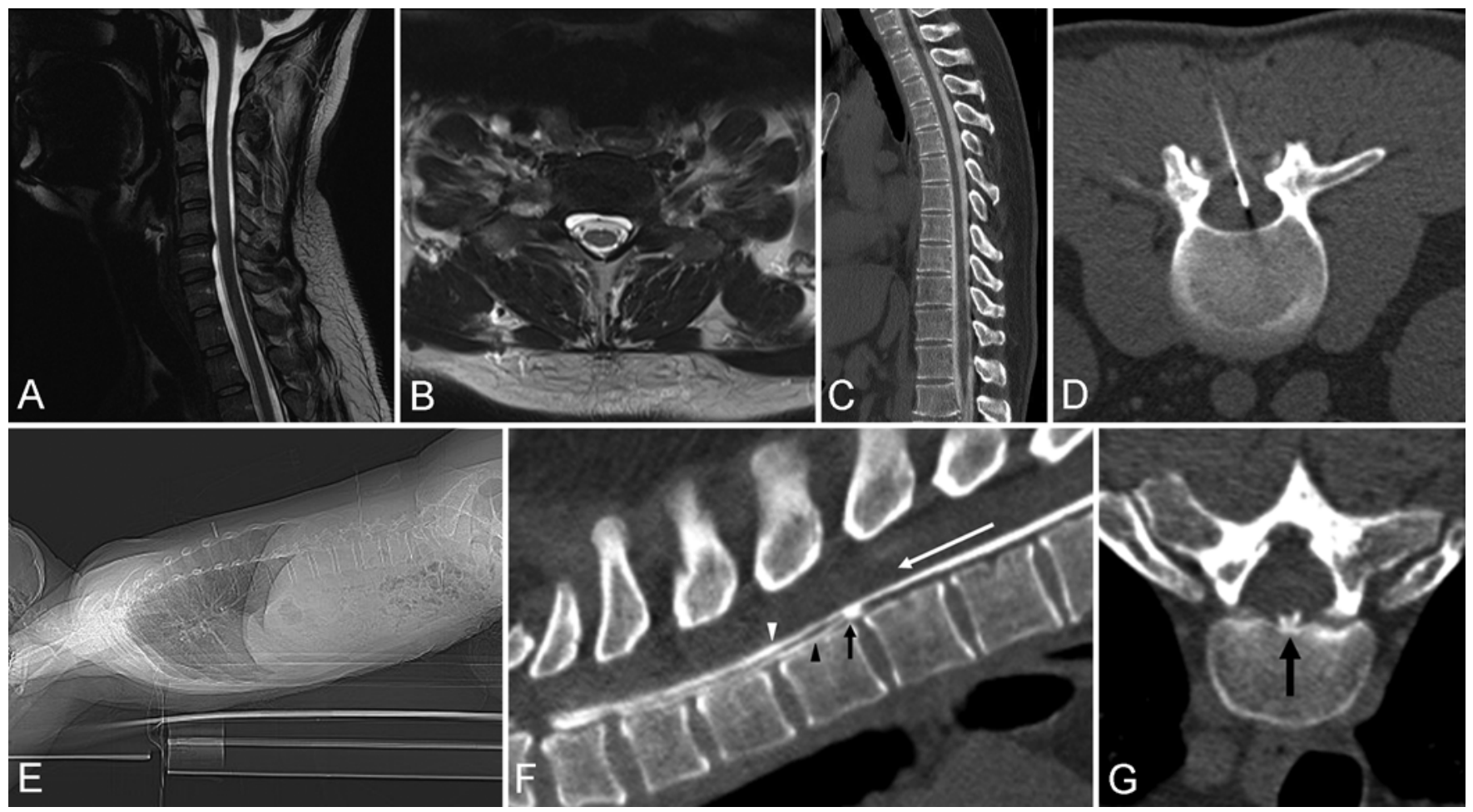

FIG. 2. Case 1. Images obtained in a 37-year-old man with a high-flow CSF leak caused by a spiculated osteophyte piercing the dura at the T2-3 interspace. A and B: Sagittal (A) and axial (B) T2-weighted MR images of the spine demonstrating the ventral epidural fluid collection and scattered osteophytes. C: Conventional myelogram demonstrating diffuse ventral epidural contrast, consistent with a spinal CSF leak. D: Localized CT scan at the time of ultrafast dynamic CT myelography confirming the position of the needle tip in the center of the spinal canal. E: Lateral scout image obtained during patient positioning for the ultrafast CT myelogram confirming that contrast should flow downhill from the tip of the spinal needle unimpeded in a cephalad direction, without dependent pooling in the thoracic spine or upper lumbar spine. F: Reconstructed sagittal ultrafast dynamic CT myelography image demonstrating an immediate fast leak into the ventral epidural space at the T2-3 interspace caused by a small spiculated osteophyte piercing the dura. The white arrow indicates the direction of contrast flow during ultrafast dynamic CT myelography. The black arrow indicates the precise location of the leak with the associated spiculated osteophyte and splitting of the contrast column into subarachnoid and epidural contrast flowing cephalad from the hole in the dura. The black arrowhead shows the portion of contrast flowing in a cephalad direction within the epidural space cranial to the leak location. The white arrowhead shows the portion of contrast flowing in a cephalad direction within the subarachnoid space cranial to the leak location. G: Precontrast CT image demonstrating the bilobed spiculated appearance of the implicated osteophyte (arrow) located in the midline at the T2-3 interspace.

\section{Discussion}

In this study we demonstrate that ultrafast dynamic CT myelography can accurately define the precise location of high-flow fast spinal CSF leaks caused by spiculated osteophytes of the spine piercing the dura. These findings are important because the precise localization of the leak site, in addition to a clear understanding of the underlying cause of the leak, is necessary to provide targeted treatment via percutaneous or surgical methods.

This study is also important because it demonstrates that spinal osteophytes violating the dura are a more frequent cause of high-flow CSF leaks than previously recognized. This pathophysiology is similarly supported by other studies that have suggested calcified disc extrusions may also be the underlying cause of dural rents resulting in ventral transdural cord herniation..$^{2,10}$ We have shown that the vast majority $(86 \%)$ of the fast leaks caused by spiculated osteophytes occur between C-5 and T-7. This distribution is anatomically consistent with the portion of the dura that is most closely opposed and draped over the dorsal surface of the intervertebral discs and vertebral bodies secondary to the normal spinal curvature. As the ventral dura is draped over and stretched against the spiculated osteophytes, an environment is created allowing for erosion and puncture of the calcified spicule through the dura (Fig. 4). Understanding of this pathophysiology may allow for advancements in percutaneous or surgical therapies aimed at treating the cause of this type of high-flow CSF leak.

Previous authors have described techniques aimed at improving both the spatial and temporal resolution required to identify and localize the site of high-flow leaks. ${ }^{5,6}$ Our study adds to the current literature by demonstrating in a large group of patients that the ultrafast dynamic CT myelography technique provides the spatial and temporal resolution required to identify the precise location of fast high-flow CSF leaks. In addition, with this precise local- 

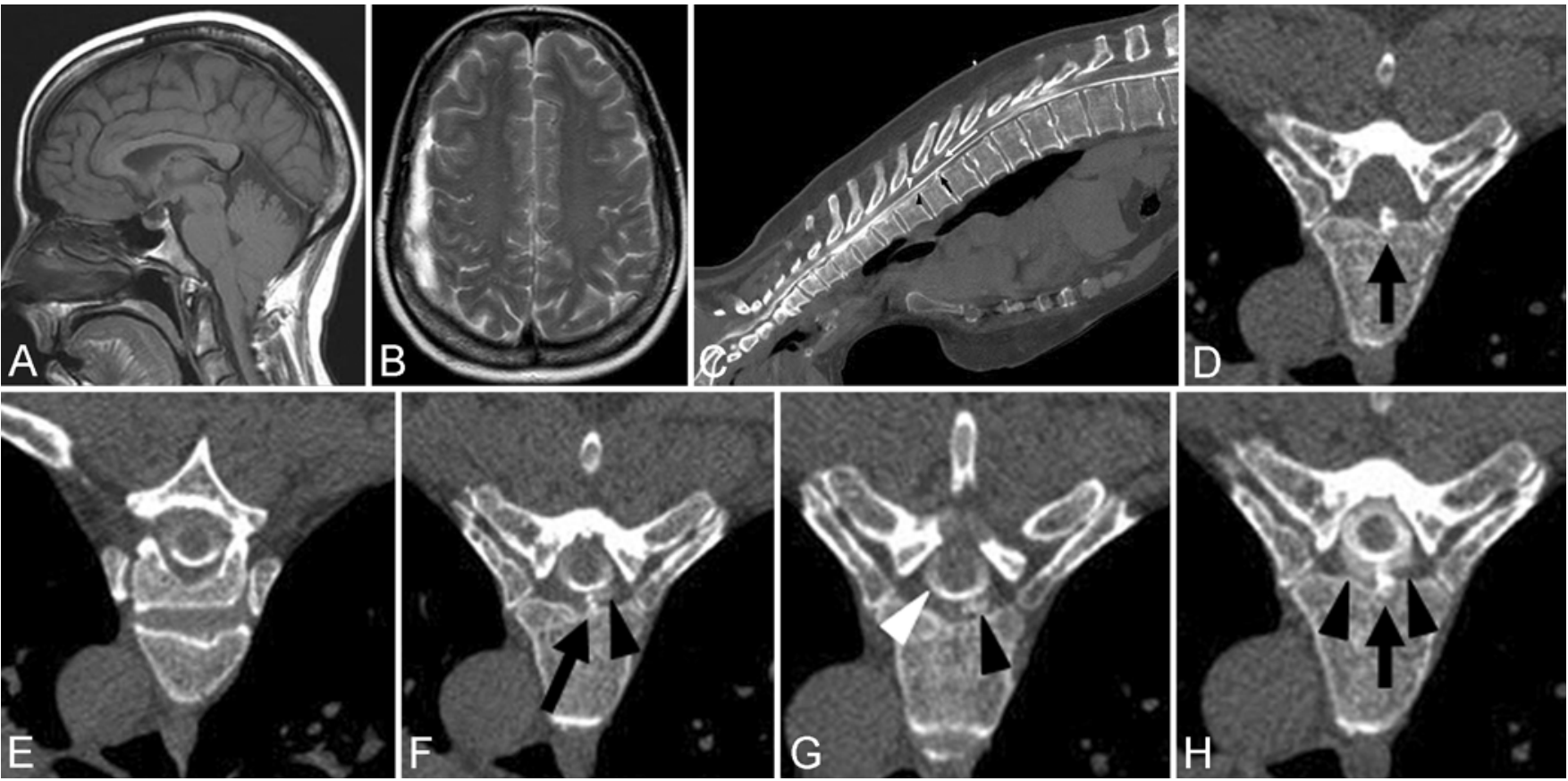

FIG. 3. Case 2. Images obtained in a 51-year-old woman with a high-flow fast CSF leak caused by a spiculated osteophyte piercing the ventral dura slightly to the right of midline at the T5-6 interspace. A and B: Sagittal T1-weighted (A) and axial T2-weighted (B) fast spin echo MR brain images demonstrating sagging of the brain and subdural fluid collections consistent with low-pressure headache syndrome. C: Sagittal reformatted ultrafast CT myelographic image of an early CT series acquisition demonstrating the fast CSF leak at the T5-6 interspace where a sharp osteophyte or calcified disc remnant pierces the dura. The white arrow indicates the direction of contrast flow during ultrafast dynamic CT myelography. The black arrow indicates the precise location of the leak with associated spiculated osteophyte and splitting of the contrast column into subarachnoid and epidural contrast, flowing cephalad from the hole in the dura. The black arrowhead demonstrates a portion of contrast flowing in a cephalad direction within the epidural space cranial to the leak location. The white arrowhead demonstrates a portion of contrast flowing in a cephalad direction within the subarachnoid space cranial to the leak location. D: Axial precontrast CT scan demonstrating the appearance of the implicated osteophyte (arrow). E: Axial CT scan just caudal to the leak site obtained 20 seconds following initiation of contrast injection. Contrast is only present within the subarachnoid space. F: Axial CT scan at the site of the leak and associated osteophyte obtained 20 seconds following initiation of contrast injection. Contrast is noted spilling into the epidural space (arrowhead) adjacent to the osteophyte (arrow). G: Axial CT scan just cranial and downstream to the leak site obtained 20 seconds following initiation of contrast injection, demonstrating both the contrast within the subarachnoid space (white arrowhead) as well as the second column of contrast more ventrally within the ventral epidural space (black arrowhead). H: Axial CT scan at the site of the leak obtained 55 seconds following the initiation of contrast injection. Note the rapid accumulation of contrast within a ventral epidural fluid collection (arrowheads). The arrow indicates the precise location of the leak with an associated spiculated osteophyte.

ization information we were able to identify small spiculated osteophytes piercing the dura as a frequent cause of these leaks. These small spicules are described as osteophytes, but may represent the calcified remnants of small disc extrusions. All of the causative osteophytes in our series were small. There were no causative large broadbased osteophytes in this series. Presumably, large broadbased osteophytes push the dura away without piercing the dura, while small spiculated osteophytes are capable of puncturing or eroding through the dura.

Precise localization of high-flow fast CSF leaks is necessary to direct appropriate therapy. Knowledge that a small spiculated osteophyte has eroded through or pierced the dura may impact the likelihood that treatment with a percutaneous blood patch will plug the leak. The precise localization of the leak is also very important because once contrast has leaked out through the hole in the dura it will travel out into the epidural fat in the neural foramina, or pool in extradural locations quite remote from the ac- tual leak site. Without the immediate temporal resolution of ultrafast dynamic CT myelography, the radiologist is at significant risk of incorrectly attributing the pooled contrast in the neural foramina or other remote locations as the site of leak. The incorrect localization can then lead to misdirected treatment via percutaneous or surgical methods. It is important for the treating physician to recognize that many of these patients had multiple sharp spurs, making it difficult to anticipate which spur was actually piercing the dura and causing the leak. In our experience we were unable to accurately prognosticate which spicule was causing the leak based on their appearance alone. It was only after obtaining the ultrafast dynamic CT myelogram that the implicated osteophyte could be confidently determined.

Digital subtraction myelography has previously been shown to be a useful adjunct in the localization of fast high-flow spinal CSF leaks. ${ }^{3}$ The ultrafast dynamic CT myelography technique has several potential advantages 

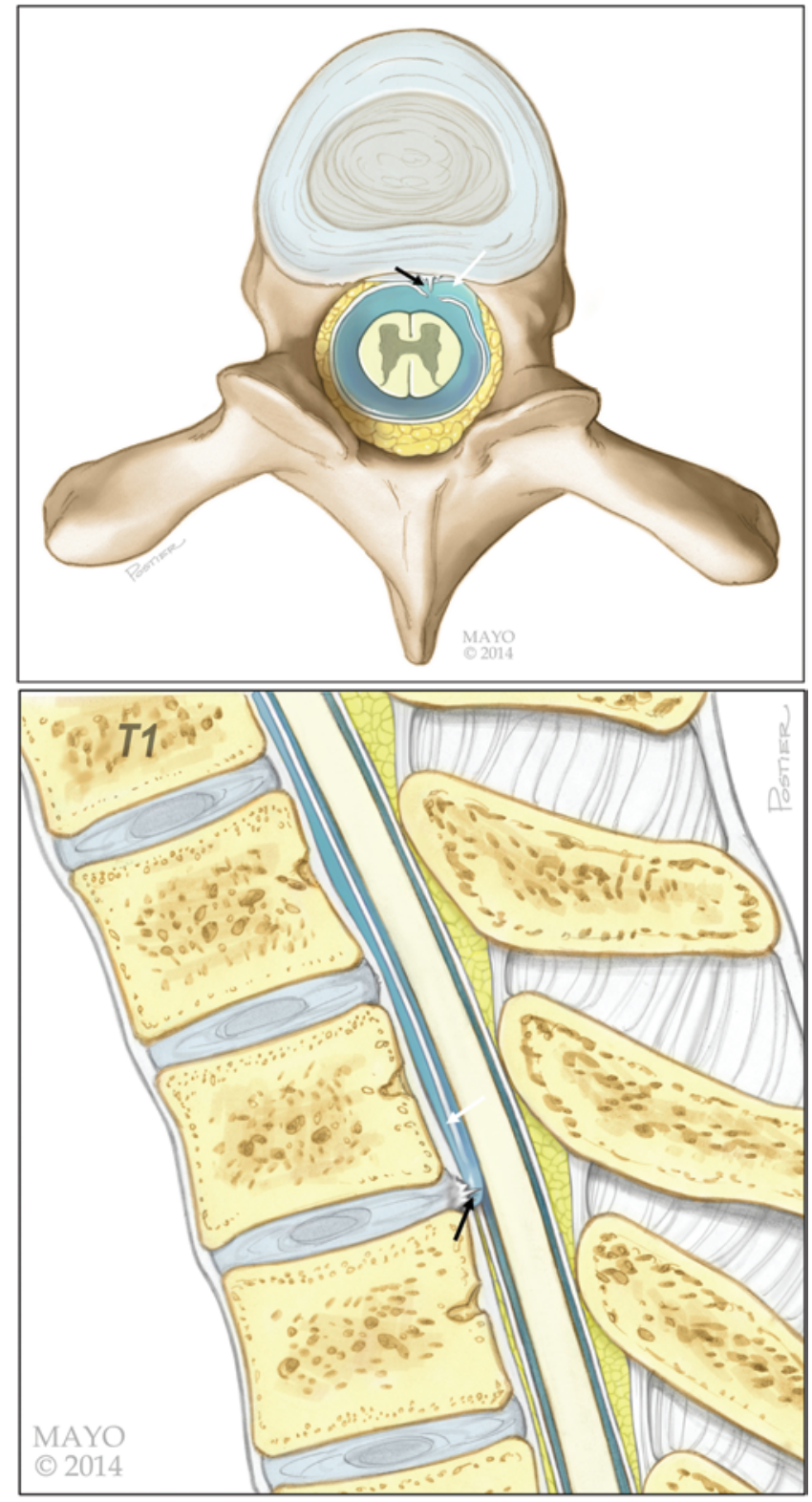

FIG. 4. Illustration of a typical high-flow CSF leak caused by a calcified spicule piercing the dura at the T3-4 interspace. Axial (upper) and sagittal (lower) illustrations of the spine demonstrating the ventral epidural fluid collection/leak associated (white arrow) with the small spicule piercing the dura (black arrow). Used with permission from the Mayo Foundation for Medical Education and Research.

over DS myelography. First, the anatomical detail and spatial resolution with the CT technique allows not only the precise localization of the leak site but also identification of the causative associated spiculated osteophytes. Second, this technique allows more extensive coverage of the spine without the limited field of view of the DS myelography technique. The CT technique is not subject to the limitations of body habitus in the shoulder region that can be encountered with DS myelography. Lastly, the CT technique is less constrained by the timing limitations that are encountered with a single-acquisition DS myelogram.
The relative disadvantages of the $\mathrm{CT}$ technique include potentially more required $\mathrm{CT}$ series acquisitions, and thus more radiation dose, as well as increased complexity of patient positioning in the CT gantry. Many of these patients undergo multiple conventional CT myelograms over the years that fail to identify the CSF leak and lead to significant delay in diagnosis. Application of the ultrafast CT myelography technique more routinely in the appropriate clinical setting may allow for a net radiation exposure reduction. The ultrafast CT myelography technique described remains the technique currently used at our institution for the evaluation of fast high-flow CSF leaks.

Our study has several limitations. The study was conducted retrospectively; thus, it was subject to bias. Although to our knowledge this study is the largest of this specific group of patients with high-flow fast CSF leaks imaged with ultrafast CT myelography, it still involved a relatively small group of patients. In addition, the ultrafast CT myelograms were not directly compared with DS myelography. This study provides good evidence that ultrafast CT myelography can be a useful adjunct in the evaluation of patients with high-flow fast spinal CSF leaks.

\section{Conclusions}

Spinal osteophytes violating the dura are a more frequent cause of high-flow CSF leaks than previously recognized. Ultrafast dynamic CT myelography adds value beyond standard dynamic myelography or DS myelography in the diagnosis and anatomical characterization of high-flow spinal CSF leaks caused by these osteophytes. This information allows for appropriate planning for percutaneous or surgical treatment. Ultrafast dynamic CT myelography provides the spatial and temporal resolution necessary to diagnose and localize spiculated osteophytes, which are a frequent cause of high-flow spinal CSF leaks.

\section{Acknowledgments}

We thank Debra Hanson from Research and Academic Support Services at Mayo Clinic for her help in preparing the manuscript and Jim Postier for the preparation of the medical illustrations.

\section{References}

1. Akbar JJ, Luetmer PH, Schwartz KM, Hunt CH, Diehn FE, Eckel LJ: The role of MR myelography with intrathecal gadolinium in localization of spinal CSF leaks in patients with spontaneous intracranial hypotension. AJNR Am J Neuroradiol 33:535-540, 2012

2. Brus-Ramer M, Dillon WP: Idiopathic thoracic spinal cord herniation: retrospective analysis supporting a mechanism of diskogenic dural injury and subsequent tamponade. AJNR Am J Neuroradiol 33:52-56, 2012

3. Hoxworth JM, Patel AC, Bosch EP, Nelson KD: Localization of a rapid CSF leak with digital subtraction myelography. AJNR Am J Neuroradiol 30:516-519, 2009

4. Inamasu J, Guiot BH: Intracranial hypotension with spinal pathology. Spine J 6:591-599, 2006

5. Kumar N, Lindell EP, Wilden JA, Davis DH: Role of dynamic CT myelography in identifying the etiology of superficial siderosis. Neurology 65:486-488, 2005 
6. Luetmer PH, Mokri B: Dynamic CT myelography: a technique for localizing high-flow spinal cerebrospinal fluid leaks. AJNR Am J Neuroradiol 24:1711-1714, 2003

7. Luetmer PH, Schwartz KM, Eckel LJ, Hunt CH, Carter RE, Diehn FE: When should I do dynamic CT myelography? Predicting fast spinal CSF leaks in patients with spontaneous intracranial hypotension. AJNR Am J Neuroradiol 33:690 694, 2012

8. Maher CO, Meyer FB: Surgical treatment of nonatherosclerotic lesions of the extracranial carotid artery. Neurosurg Clin N Am 11:309-322, 2000

9. Mokri B: Spontaneous cerebrospinal fluid leaks: from intracranial hypotension to cerebrospinal fluid hypovolemia-evolution of a concept. Mayo Clin Proc 74:1113-1123, 1999

10. Sagiuchi T, Iida H, Tachibana S, Utsuki S, Tanaka R, Fujii $\mathrm{K}$ : Idiopathic spinal cord herniation associated with calcified thoracic disc extrusion-case report. Neurol Med Chir (Tokyo) 43:364-368, 2003

11. Schievink WI: Spontaneous spinal cerebrospinal fluid leaks and intracranial hypotension. JAMA 295:2286-2296, 2006

12. Schievink WI, Palestrant D, Maya MM, Rappard G: Spontaneous spinal cerebrospinal fluid leak as a cause of coma after craniotomy for clipping of an unruptured intracranial aneurysm. J Neurosurg 110:521-524, 2009

\section{Author Contributions}

Conception and design: Thielen, Morris. Analysis and interpretation of data: Thielen, Sillery, Yu. Drafting the article: Thielen, Sillery, Hoxworth, Diehn, Wald, Rosebrock, Luetmer. Approved the final version of the manuscript on behalf of all authors: Thielen.

\section{Supplemental Information}

Previous Presentation

Results were presented at the 49th Annual Meeting of the American Society of Neuroradiology in Seattle, Washington, on June 6, 2011.

\section{Correspondence}

Kent R. Thielen, Department of Radiology, Mayo Clinic, 200 1st St. SW, Rochester, MN 55905. email: thielen.kent@mayo.edu. 